\title{
COMPLEX SYMMETRIC TRIANGULAR OPERATORS
}

\begin{abstract}
SEN ZHU
Abstract. In this paper we explore complex symmetric operators with eigenvalues. We develop new techniques to give a geometric description of certain complex symmetric triangular operators. This extends a recent result of L. Balayan and S. Garcia concerning finite-dimensional complex symmetric operators. On the other hand, using Apostol's triangular representation for Hilbert space operators, we give a description of the internal structure of complex symmetric operators.
\end{abstract}

Mathematics subject classification (2010): Primary 47A66, 47B99; Secondary 47A05.

Keywords and phrases: Complex symmetric operators, triangular operators, bitriangular operators, triangular representation, Cowen-Douglas operators.

\section{REFERENCES}

[1] C. Apostol And B. B. Morrel, On uniform approximation of operators by simple models, Indiana Univ. Math. J. 26, 3 (1977), 427-442.

[2] L. BALAYAN AND S. R. GARCIA, Unitary equivalence to a complex symmetric matrix: geometric criteria, Oper. Matrices 4, 1 (2010), 53-76.

[3] N. Chevrot, E. Fricain, AND D. Timotin, The characteristic function of a complex symmetric contraction, Proc. Amer. Math. Soc. 135, 9 (2007), 2877-2886 (electronic).

[4] M. J. Cowen And R. G. Douglas, Complex geometry and operator theory, Acta Math. 141, 3-4 (1978), 187-261.

[5] K. R. Davidson And D. A. Herrero, The Jordan form of a bitriangular operator, J. Funct. Anal. 94, 1 (1990), 27-73.

[6] S. R. GARCiA, The norm and modulus of a Foguel operator, Indiana Univ. Math. J. 58, 5 (2009), 2305-2316.

[7] S. R. GARCIA AND D. E. PoORE, On the norm closure of the complex symmetric operators: compact operators and weighted shifts, J. Funct. Anal. 264, 3 (2013), 691-712.

[8] S. R. GARCIA, D. E. POORE, AND J. E. TENER, Unitary equivalence to a complex symmetric matrix: low dimensions, Linear Algebra Appl. 437, 1 (2012), 271-284.

[9] S. R. GARCIA, D. E. POORE, AND M. WYSE, Unitary equivalence to a complex symmetric matrix: a modulus criterion, Oper. Matrices 2, 5 (2011), 273-287.

[10] S. R. Garcia AND M. PutinaR, Complex symmetric operators and applications, Trans. Amer. Math. Soc. 358, 3 (2006), 1285-1315 (electronic).

[11] S. R. Garcia And M. Putinar, Complex symmetric operators and applications. II, Trans. Amer. Math. Soc. 359, 8 (2007), 3913-3931 (electronic).

[12] S. R. GARCIA AND W. Ross, Recent progress on truncated Toeplitz operators, Fields Institute Communications 65 (2013), 275-319.

[13] S. R. GARCia, W. Ross, AND W. R. Wogen, $C^{*}$-algebras generated by truncated Toeplitz operators, Oper. Theory. Adv. Appl. 236 (2013), 181-192.

[14] S. R. GARCIA AND J. E. TENER, Unitary equivalence of a matrix to its transpose, J. Operator Theory 68, 1 (2012), 179-203.

[15] S. R. Garcia And W. R. Wogen, Complex symmetric partial isometries, J. Funct. Anal. 257, 4 (2009), 1251-1260.

[16] S. R. Garcia AND W. R. Wogen, Some new classes of complex symmetric operators, Trans. Amer. Math. Soc. 362, 11 (2010), 6065-6077. 
[17] T. M. Gilbreath AND W. R. Wogen, Remarks on the structure of complex symmetric operators, Integral Equations Operator Theory 59, 4 (2007), 585-590.

[18] P. R. Halmos, A Hilbert Space Problem Book, Second edition. Graduate Texts in Mathematics, 19. Encyclopedia of Mathematics and its Applications, 17. Springer-Verlag, New York-Berlin, 1982.

[19] D. A. Herrero, Spectral pictures of operators in the Cowen-Douglas class $B_{n}(\Omega)$ and its closure, J. Operator Theory 18, 2 (1987), 213-222.

[20] D. A. HERRERO, Most quasitriangular operators are triangular, most biquasitriangular operators are bitriangular, J. Operator Theory 20, 2 (1988), 251-267.

[21] D. A. HerRero, Approximation of Hilbert space operators. Vol. 1, 2nd Edition, Vol. 224 of Pitman Research Notes in Mathematics Series, Longman Scientific \& Technical, Harlow, 1989.

[22] D. A. Herrero, Triangular operators, Bull. London Math. Soc. 23, 6 (1991), 513-554.

[23] D. SARASON, Algebraic properties of truncated Toeplitz operators, Oper. Matrices 1, 4 (2007), $491-$ 526.

[24] S. ZHU, Approximate unitary equivalence to skew symmetric operators, Complex Analysis and Operator Theory 8, 7 (2014), 1565-1580.

[25] S. ZHU AND C. G. LI, Complex symmetry of a dense class of operators, Integral Equations Operator Theory 73, 2 (2012), 255-272.

[26] S. Zhu And C. G. LI, Complex symmetric weighted shifts, Trans. Amer. Math. Soc. 365, 1 (2013), $511-530$. 